Pacific Journal of Mathematics

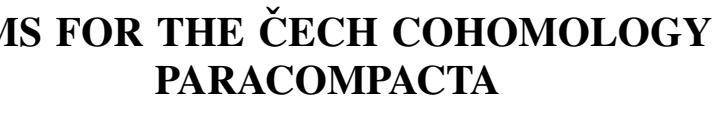




\title{
AXIOMS FOR THE ČECH COHOMOLOGY OF PARACOMPACTA
}

\author{
PHILIP BACON
}

\begin{abstract}
The Čech cohomology of paracompact Hausdorff spaces is characterized (up to isomorphism) by supplementing the Eilenberg-Steenrod axioms for cohomology.
\end{abstract}

1. The theorem. Let $C$ be an admissible category for homology theory, as defined by Eilenberg-Steenrod [2, p. 5]. We say $H$ is a cohomology theory on $C$ if $H$ satisfies the Eilenberg-Steenrod axioms [2, p. 14] and (1.1) and (1.2) below.

(1.1) Additivity axiom. If a space $X$ is the union of a collection $\mathscr{Q}$ of pairwise disjoint open sets and for each $U$ in $\mathscr{C}$ the inclusion map $j_{U}: U \subset X$ is in $C$, then $\left\{j_{U}^{*}: H^{q}(X) \rightarrow H^{q}(U) \mid U \in \mathscr{C}\right\}$ is a representation of $H^{q}(X)$ as a direct product.

(1.2) Nonnegativity axiom. $\quad H^{q}(X, A)=0$ if $(X, A) \in C$ and $q<0$.

We say a cohomology theory $H$ is point reductive if (1.3) holds. The Čech and Alexander-Spanier cohomology theories are examples of point reductive cohomology theories.

(1.3) If $X \in C$, if $S$ is a singleton (one-point) subset of $X$, if $h \in$ $H^{q}(X)$ and if $h \mid S=0$, then there is a neighborhood $N$ of $S$ such that the inclusion map $N \subset X$ is in $C$ and $h \mid N=0$.

A homomorphism $t: H \rightarrow J$ of cohomology theories $H$ and $J$ on $C$ is a natural transformation from $H$ to $J$ that commutes with coboundary homomorphisms. A pair $(X, A)$ is a paracompact pair if $X$ is a paracompact Hausdorff space and $A$ is a closed subset of $X$. The category $\boldsymbol{P}$ of paracompact pairs and all maps among them is an admissible category for homology theory. We shall prove-

Theorem 1. Suppose $H$ and $L$ are cohomology theories on $\boldsymbol{P}, \boldsymbol{S}$ is a singleton, $k^{0}(S): H^{0}(S) \rightarrow L^{0}(S)$ is a homomorphism and $H$ is point reductive. There is a unique extension of $k^{0}(S)$ to a homomorphism $k: H \rightarrow L$ of cohomology theories. If $L$ is point reductive and $k^{0}(S)$ is an isomorphism, then $k$ is an isomorphism.

For related theorems see [1], [2, p. 287, Theorem 12.1], and [6]. 
2. The proof. As an immediate consequence of a theorem due to Lawson [3] we have the following.

LeMma 2.1. Suppose $J$ and $H$ are point reductive cohomology theories on $\boldsymbol{P}$ and $m: J \rightarrow H$ is a homomorphism of cohomology theories such that $m^{0}(S): J^{0}(S) \rightarrow H^{0}(S)$ is an isomorphism for some singleton $S$. Then $m$ is an isomorphism of cohomology theories.

A polyhedron is the union of the simplexes of a geometric simplicial complex with the metric topology [2, p. 75]. A polyhedron and its underlying simplicial complex will be denoted by the same symbol. If $L$ is a subcomplex of a simplicial complex $K,(K, L)$ is a polyhedral pair. The category $\boldsymbol{K}$ of polyhedral pairs and all maps among them is an admissible category for homology theory. It is a subcategory of $\boldsymbol{P}$.

If $H$ is a cohomology theory on $K$, the Čech method may be applied to $H$ to define a cohomology theory $J$ on $P$. $J$ is called the Cech extension of $H$. We briefly recall the method (see [4]). Let $(X, A)$ be a paracompact pair and let $\Lambda(X)$ be the collection of all locally finite open covers of $X$. If $\alpha \in \Lambda(X)$, let $\left(X_{\alpha}, A_{\alpha}\right)$ be the polyhedral pair determined by the nerve of $\alpha$. If $\beta \in \Lambda(X)$ and $\beta$ refines $\alpha$, there is a simplicial map $r_{\alpha \beta}:\left(X_{\beta}, A_{\beta}\right) \rightarrow\left(X_{\alpha}, A_{\alpha}\right)$ that maps each vertex $V$ of $X_{\beta}$ to a vertex $U$ of $X_{\alpha}$ such that $V \subset U$. If in addition $\gamma \in$ $\Lambda(X)$ and $\gamma$ refines $\beta, r_{\alpha \beta} r_{\beta \gamma}$ and $r_{\alpha \gamma}$ are homotopic, which implies that $r_{\beta \gamma}^{*} r_{\alpha \beta}^{*}=r_{\alpha \gamma}^{*}$. Hence there is a direct system of groups $\left\{H^{q}\left(X_{\alpha}, A_{\alpha}\right) \mid \alpha \epsilon\right.$ $\Lambda(X)\}$ and homomorphisms $\left\{r_{\alpha \beta}^{*} \mid \beta\right.$ refines $\left.\alpha\right\}$, whose direct limit we shall denote by $J^{q}(X, A)$. The coboundary homomorphism $\delta: J^{q}(A) \rightarrow$ $J^{q+1}(X, A)$ for a paracompact pair $(X, A)$ and the homomorphism $J^{q}(f)$ : $J^{q}(Y, B) \rightarrow J^{q}(X, A)$ induced by a map $f:(X, A) \rightarrow(Y, B)$ in $\boldsymbol{P}$ are suitable limit homomorphisms.

LEMmA 2.2. If $H$ is a cohomology theory on $K$, then the Čech extension of $H$ is a point reductive cohomology theory on $\boldsymbol{P}$.

The proof is left to the reader.

Suppose $H$ is a cohomology theory on $\boldsymbol{P}, L$ is the restriction of $H$ to $\boldsymbol{K}$ and $J$ is the Čech extension of $L$. We shall construct a homomorphism $m: J \rightarrow H$ called the canonical homomorphism.

Let $(X, A)$ be a paracompact pair and let $\Lambda(X)$ be the collection of locally finite open covers of $X$. If $\alpha \in \Lambda(X)$, there is a map $r_{\alpha}:(X$, $A) \rightarrow\left(X_{\alpha}, A_{\alpha}\right)$ defined by a partition of unity subordinate to $\alpha[5, \mathrm{p}$. 833, Proposition 2]. Any two choices of $r_{\alpha}$ are homotopic. If $\beta \in$ $\Lambda(X)$ and $\beta$ refines $\alpha$, then $r_{\alpha \beta} r_{\beta}$ and $r_{\alpha}$ are homotopic, which implies that $\boldsymbol{r}_{\beta}^{*} \boldsymbol{r}_{\alpha \beta}^{*}=r_{\alpha}^{*}$. Hence the homomorphisms $\left\{H^{q}\left(r_{\alpha}\right) \mid \alpha \in \Lambda(X)\right\}$ induce 
a homomorphism $m^{q}(X, A): J^{q}(X, A) \rightarrow H^{q}(X, A)$. It follows from the way $m$ is defined that $m$ is a homomorphism from $J$ to $H$.

If $S$ is a singleton, $m^{0}(S): J^{0}(S) \rightarrow H^{0}(S)$ is an isomorphism because $\left\{r_{\alpha} \mid \alpha \in \Lambda(S)\right\}$ consists of just one map, a homeomorphism. Hence by Lemmas 2.1 and 2.2 we have-

LeMMA 2.3. If $H$ is a point reductive cohomology theory on $\boldsymbol{P}$ and $J$ is the Cech extension of the restriction of $H$ to $\boldsymbol{K}$, then the canonical homomorphism $m: J \rightarrow H$ is an isomorphism.

Lemma 2.3 implies Lemma 2.4, which in turn implies Lemma 2.5.

LEMma 2.4. If $H$ is a point reductive cohomology theory on $\boldsymbol{P}$ and $(X, A) \in \boldsymbol{P}$, then $\left(H^{q}(X, A),\left\{H^{q}\left(r_{\alpha}\right) \mid \alpha \in \Lambda(X)\right\}\right)$ is a direct limit of the direct system $\left(\left\{H^{q}\left(X_{\alpha}, A_{\alpha}\right)\right\},\left\{H^{q}\left(r_{\alpha \beta}\right)\right\}\right)$.

Lemma 2.5. Suppose $H$ and $L$ are cohomology theories on $\boldsymbol{P}$ and $t: H|\boldsymbol{K} \rightarrow L| \boldsymbol{K}$ is a homomorphism of cohomology theories. If $H$ is point reductive, there is a unique extension of $t$ to a homomorphism $k: H \rightarrow L$.

Lemma 2.6 was essentially proved by Milnor in [6], although the uniqueness part of it was not stated there.

Lemma 2.6. Suppose $H$ and $J$ are cohomology theories on $\boldsymbol{K}$. If $S$ is a singleton and $t^{0}(S): H^{\circ}(S) \rightarrow J^{0}(S)$ is a homomorphism, there is a unique extension of $t^{0}(S)$ to a homomorphism $t: H \rightarrow J$ of cohomology theories on $\boldsymbol{K}$.

Theorem 1 follows from Lemmas 2.5 and 2.6.

\section{REFERENCES}

1. C. H. Dowker, Homology groups of relations, Ann. of Math., 56 (1952), 84-95.

2. S. Eilenberg and N. Steenrod, Foundations of Algebraic Topology, Princeton University Press, Princeton, N. J., 1952.

3. J. P. Lawson, Comparison of taut cohomologies, Aequationes Math., 2 (1973), 201209.

4. C. N. Lee and F. A. Raymond, Ц̌ech extensions of contravariant functors, Trans. Amer. Math. Soc., 133 (1968), 415-434.

5. E. Michael, A note on paracompact spaces, Proc. Amer. Math. Soc., 4 (1953), 831838.

6. J. Milnor, On axiomatic homology theory, Pacific J. Math., 12 (1962), 337-341.

Received November 13, 1972 and in revised form October 24, 1973.

UNIVERSITY OF FLORIDA 



\section{PACIFIC JOURNAL OF MATHEMATICS}

EDITORS

RICHARD ARENS (Managing Editor)

University of California

Los Angeles, California 90024

R. A. Beaumont

University of Washington

Seattle, Washington 98105

J. DugundJI

Department of Mathematics

University of Southern California

Los Angeles, California 90007

D. Gilbarg and J. Milgram

Stanford University

Stanford, California 94305

\section{ASSOCIATE EDITORS}

E. F. BECKENBACH

B. H. NeumanN

F. WOLF

K. YOSHIDA

\section{SUPPORTING INSTITUTIONS}

UNIVERSITY OF BRITISH COLUMBIA CALIFORNIA INSTITUTE OF TECHNOLOGY

UNIVERSITY OF CALIFORNIA

MONTANA STATE UNIVERSITY

UNIVERSITY OF NEVADA

NEW MEXICO STATE UNIVERSITY

OREGON STATE UNIVERSITY

UNIVERSITY OF OREGON

OSAKA UNIVERSITY

\author{
UNIVERSITY OF SOUTHERN CALIFORNIA \\ STANFORD UNIVERSITY \\ UNIVERSITY OF TOKYO \\ UNIVERSITY OF UTAH \\ WASHINGTON STATE UNIVERSITY \\ UNIVERSITY OF WASHINGTON \\ $\stackrel{*}{*} \stackrel{*}{*}{ }^{*}{ }^{*}{ }^{2}$ AMERICAN MATHEMATICAL SOCIETY \\ NAVAL WEAPONS CENTER
}




\section{Pacific Journal of Mathematics}

\section{Vol. 52, No. $1 \quad$ January, 1974}

David R. Adams, On the exceptional sets for spaces of potentials ............ 1

Philip Bacon, Axioms for the Čech cohomology of paracompacta ............ 7

Selwyn Ross Caradus, Perturbation theory for generalized Fredholm operators ..... 11

Kuang-Ho Chen, Phragmén-Lindelöf type theorems for a system of nonhomogeneous equations ............................ 17

Frederick Knowles Dashiell, Jr., Isomorphism problems for the Baire classes .......

M. G. Deshpande and V. K. Deshpande, Rings whose proper homomorphic images are right subdirectly irreducible . . . . . . . . . . . . . . . . . . . . . . . . .

Mary Rodriguez Embry, Self adjoint strictly cyclic operator algebras .............

Paul Erdős, On the distribution of numbers of the form $\sigma(n) / n$ and on some related

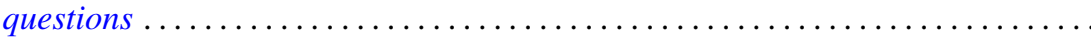

Richard Joseph Fleming and James E. Jamison, Hermitian and adjoint abelian

operators on certain Banach spaces ............................

Stanley P. Gudder and L. Haskins, The center of a poset .................. 85

Richard Howard Herman, Automorphism groups of operator algebras . . . ........

Worthen N. Hunsacker and Somashekhar Amrith Naimpally, Local compactness of families of continuous point-compact relations ....................

Donald Gordon James, On the normal subgroups of integral orthogonal groups ....

Eugene Carlyle Johnsen and Thomas Frederick Storer, Combinatorial structures in

loops. II. Commutative inverse property cyclic neofields of prime-power

order.

Ka-Sing Lau, Extreme operators on Choquet simplexes . . . . . . . . . . . . . . 129

Philip A. Leonard and Kenneth S. Williams, The septic character of 2, 3, 5 and $7 \ldots 143$

Dennis McGavran and Jingyal Pak, On the Nielsen number of a fiber map ........ 149

Stuart Edward Mills, Normed Köthe spaces as intermediate spaces of $L_{1}$ and

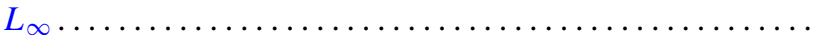

Philip Olin, Free products and elementary equivalence. .

Louis Jackson Ratliff, Jr., Locally quasi-unmixed Noetherian rings and ideals of the principal class.

Seiya Sasao, Homotopy types of spherical fibre spaces over spheres ...

Helga Schirmer, Fixed point sets of polyhedra ...

Kevin James Sharpe, Compatible topologies and continuous irreducible

representations.

Frank Siwiec, On defining a space by a weak base . . . . . . . . . . . . . . . 233

James McLean Sloss, Global reflection for a class of simple closed curves ....... 247

M. V. Subba Rao, On two congruences for primality . .

Raymond D. Terry, Oscillatory properties of a delay differential equation of even

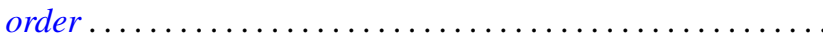

Joseph Dinneen Ward, Chebyshev centers in spaces of continuous functions . .

Robert Breckenridge Warfield, Jr., The uniqueness of elongations of Abelian

groups...

V. M. Warfield, Existence and adjoint theorems for linear stochastic differential

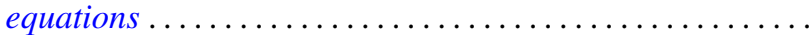

